\title{
Las comunidades de nematodos como indicadores ambientales
}

Nematode communities as environmental indicators

Ingrid Varela-Benavides'

Fecha de recepción: 17 de julio del 2013

Fecha de aprobación: I de octubre del 2013

Varela-Benavides, I. Las comunidades de nemátodos como indicadores ambientales.

Tecnología en Marcha. Número Especial. Pág 30-37.

I Bióloga. Máster en Agricultura Ecológica. Laboratorio de Nematología, Centro de Investigación y Desarrollo Agrícola Sostenible para el Trópico Húmedo, Instituto Tecnológico de Costa Rica, sede San Carlos. Costa Rica. Correo electrónico: invarela@itcr.ac.cr. 


\section{Ingrid Varela Benavides}

Ingrid Varela Benavides nació y creció en la comunidad de San Vicente de Ciudad Quesada, ubicada en las faldas del volcán Platanar, Costa Rica. Hija de una familia de campesinos, se ha interesado por la producción agrícola orgánica y la conservación del ambiente.

Obtuvo su bachillerato en el Colegio Científico de San Carlos, posteriormente se graduó como Bachiller en la Carrera de Biología Tropical en la Universidad Nacional.

En el área de la biología, ha realizado estudios de campo para la identificación de especies de árboles en la zona del Golfo Dulce y en la Península de Nicoya. También ha trabajado en la conservación ex situ de animales silvestres, especialmente con mamíferos y aves nativos de Costa Rica.
Su interés en la conservación y en la producción agrícola la llevó a realizar una Maestría en Agricultura Ecológica en la Universidad Nacional. Cuenta además con un Diplomado en Administración de Empresas de la Universidad Estatal a Distancia.

Se ha capacitado en el campo de la Nematología, con interés particular en el estudio de las comunidades de nematodos del suelo.

Actualmente trabaja como investigadora en el laboratorio de Nematología de la Escuela de Agronomía del Instituto Tecnológico de Costa Rica en la sede San Carlos. Ha participado en varios proyectos entre los que destacan: "Programa interinstitucional de investigación en biodiversidad y ecología de organismos de suelo con énfasis en sistemas de producción limpia y control biológico" y el "Diagnóstico y caracterización bioquímica y molecular de nematodos noduladores (Meloidogyne spp) en cultivos tropicales y hortícolas de Costa Rica". 


\section{Palabras clave}

Comunidades de nemátodos; evaluación de ecosistemas; índice de madurez; indicadores ambientales.

\section{Resumen}

El uso de indicadores ambientales que permitan describir el estado de un ecosistema y las presiones a las que está sometido, así como indicar los avances logrados con las prácticas de mitigación establecidas son temas de interés en la actualidad.

Entre los organismos que han sido estudiados por su potencial como indicadores ambientales se encuentran los nemátodos. Estos animales son cosmopolitas y juegan un papel importante en todos los ecosistemas; además, la comunidad de nemátodos cambia de forma predecible con las perturbaciones del ambiente. Estos cambios están correlacionados con indicadores del funcionamiento del ecosistema. Aunque existen taxones indicadores de condiciones en particular, también se han desarrollado índices que permiten describir la diversidad, la estructura de la comunidad y la sucesión ecológica. También se han creado modelos indicadores de las vías metabólicas en el ecosistema del suelo.

Estos índices y modelos se basan en las diferentes estrategias de supervivencia que tienen los taxones de nematodos, ya sean colonizadores o persistentes. Los colonizadores se consideran oportunistas de ambientes enriquecidos y los persistentes son indicadores de sistemas no perturbados. Por otro lado, con el desarrollo de modernas técnicas de secuenciación se espera poder evaluar la diversidad biológica de manera más eficiente, logrando utilizar a los nemátodos en programas de monitoreo ambiental a nivel nacional e internacional.

\section{Key words}

Nematode communities; ecosystem evaluation; maturity index; environmental indicators.

\section{Abstract}

The use of environmental indicators is a topic of current interest. It allows us to describe the condition of an ecosystem and the pressures that is being subject. And at the same time indicate the effectiveness of the mitigation practices. Nematodes are among the organisms that have been studied for their potential as environmental indicators.

These animals are cosmopolitan and play an important role in all ecosystems. Furthermore the nematode community predictably changes with the disturbances to the environment. These changes are related to indicators of ecosystems functions. There are taxa indicative of conditions in particular and indices have been developed to describe the diversity, community structure and ecological succession. Models have also been created, it are indicators of metabolic pathways in the soil ecosystem. These indices and models are based on different survival strategies that have nematodes. Colonizers nematodes are considered enrichment opportunist and persister nematodes indicate system stability. Moreover, with the development of modern sequencing techniques are expected to achieve assessing biodiversity more efficiently, making use of nematodes in environmental monitoring programs nationally and internationally. 


\section{El uso de indicadores ambientales en la evaluación de ecosistemas}

En la actualidad, el uso de indicadores para evaluar la calidad de los ecosistemas es una práctica utilizada por organizaciones a nivel global. Programas e instituciones como el Canadian Environmental Sustainability Program, United States Environmental Indicators Agency y European Environment Agency concuerdan en que en el esfuerzo orientado a reducir el deterioro ambiental es útil para plantear metas, objetivos y las formas para medir el avance de estos esfuerzos (EEA, 20I2).

Los indicadores ambientales describen el estado del ecosistema en un momento dado o el impacto de una acción en éste. De esta forma permiten identificar las amenazas al ambiente y las presiones a las que está siendo sometido e indican los avances logrados con las prácticas de mitigación (EEA, 20I2).

Idealmente, los indicadores deben dar información clave acerca de la estructura, función y composición del ecosistema, proporcionar una señal temprana de los cambios en él y ayudar a entender las causas de los problemas ambientales (Dalea y Beyerler, 200 I).

Es importante que tales indicadores sean suficientemente simples como para ser monitoreados con facilidad de manera rutinaria, pero a la vez deben ser eficientes para captar la complejidad del ecosistema e identificar posibles factores que estén causando presión ambiental, de modo que faciliten la toma de decisiones, la creación de políticas y el monitoreo de la efectividad de las políticas desarrolladas (EEA, 1999).

Según Dalea y Beyerler (200l), los indicadores ecológicos deben cumplir con los siguientes criterios: ser fácilmente medibles y ser sensibles a las presiones en el ecosistema, responder al estrés de una manera predecible y tener baja variabilidad en la respuesta, predecir los cambios que pueden evitarse con medidas de gestión y tener una respuesta conocida a las perturbaciones antropogénicas y los cambios temporales.

Algunos organismos vivos que cumplen con estas características han probado ser eficientes indicadores ambientales. Los líquenes se han utilizado exitosamente como indicadores de la contaminación del aire (Hawksworth et al., 2005). Por otro lado, muchas especies de algas son eficientes indicadoras de la eutroficación del agua (Paerl et al., 2003).

Uno de los grupos más estudiados por su utilidad como indicadores ambientales son los nematodos. Hoy en día la nematofauna en todo su conjunto, tanto terrestre como acuática, se utiliza para inferir el estado y las condiciones de las redes tróficas y las funciones en ecosistemas naturales y manejados (Ferris et al., 2012).

\section{Los nematodos como} indicadores ambientales

Los nematodos son un grupo de organismos altamente diverso, que habitan una gran cantidad de ambientes y se alimentan de gran variedad de fuentes. Entre el 80 y el $90 \%$ de todos los animales multicelulares en la Tierra son nemátodos (Bongers, 1985). Actualmente se estima que pertenecen a este filo entre 40000 y 10000000 de especies (Yeates y Boag, 2006).

Los nematodos tienen un papel importante en los procesos que constituyen la mayoría de los servicios que brindan los ecosistemas a la sociedad, tales como el suministro de alimentos, agua y aire y la regulación de plagas y enfermedades (Yeates et al., 2009)

La contribución más importante de los nematodos en el ecosistema es la distribución de los nutrientes y minerales. Los nematodos son responsables de un $30 \%$ de la mineralización del nitrógeno en el suelo, siendo este el principal servicio que prestan en el ecosistema. Otras funciones que desempeñan en las redes tróficas del suelo son: secuestrar y redistribuir minerales, carbón y energía; regular las poblaciones de organismos oportunistas a través de la depredación; servir como presas de depredadores en niveles tróficos más altos; degradar toxinas que ingresen al ambiente; influir en la composición de la comunidad vegetal y su sucesión y acelerar las tasas de descomposición (Ferris, 2010).

Gracias a que los nematodos ocurren en una gran diversidad y densidad en todos los ambientes, juegan un importante papel en las redes tróficas del suelo, son aislables e identificables y pueden ser ubicados con facilidad en grupos tróficos. Son muy buenos indicadores ambientales en ecosistemas tanto terrestres como acuáticos (Bongers, 1999). 
Además, estos animales están en contacto directo con los compuestos disueltos en las partículas de agua, por lo que reaccionan rápidamente a los disturbios pero, al mismo tiempo, cada taxón del filo Nematoda responde de forma diferente a los cambios en el ambiente, por lo que la identificación de las familias y géneros de nematodos presentes en la comunidad y la determinación de su abundancia ofrecen una buena oportunidad para evaluar la condición del ecosistema con relación al impacto de los contaminantes y otros factores estresantes, así como para monitorear los cambios en la estructura y funcionamiento de las redes tróficas del suelo (Yeates y Bongers, 1999).

El estudio de los nematodos como indicadores de la productividad, contaminación y sucesión secundaria se inició en la década de 1980. Muchos autores estudiaron las comunidades de nematodos y reconocieron su utilidad para evaluar el grado de alteración y el estado de un ecosistema (Bongers, 1990, Freckman y Ettema, 1993, Wasilewska, 1994).

Entre los nematodos existen taxones indicadores. Los diplogastéridos y rhabditidos son indicadores de hábitats eutroficados, mientras que los dorilaimidos y mononchidos son comunes en hábitats poco alterados (Jensen y Mulvey, 1968, Sánchez-Moreno et al., 2009).

También la composición de la comunidad de nematodos es un buen indicador. Las alteraciones como la deforestación, la liberación de agroquímicos y desechos industriales y urbanos al ambiente, el arado y el cultivo del suelo afectan la riqueza de especies, la estructura trófica y el estado de sucesión de la comunidad de nematodos. Estos cambios están correlacionados con indicadores del funcionamiento del ecosistema, como el aumento de nitratos y fósforo en el suelo, la disminución del carbono y la biomasa microbiana y el cambio en la estructura de las redes tróficas (Culman et al., 20 l0).

También se está utilizando a los nematodos en estudios sobre el cambio climático. El aumento atmosférico del $\mathrm{CO}_{2}$, la concentración de nitrógeno y los cambios en los regímenes de precipitación, provocados por el calentamiento global, afectan la comunidad de nemátodos de una forma predecible, provocando una simplificación taxonómica y funcional de las comunidades en el suelo (Eisenhauer et al., 20।2).
Con el fin de simplificar la información que brinda el análisis de las comunidades de nematodos, se utilizan índices que permiten resumir los datos en una $\mathrm{o}$ dos variables. Los índices más comúnmente utilizados son los que determinan la diversidad, la riqueza de especies y su distribución. Estos se han complementado con el análisis de múltiples variables (Neher y Darby, 2009).

Por otro lado, se han desarrollado índices específicos para la comunidad de nematodos; estos son modelos que expresan la estructura de la comunidad y también constituyen una forma de medir la sucesión ecológica. Bongers fue el primer nematólogo en proponer el índice de madurez, que ha sido perfeccionado desde entonces (Bongers, 1990, Bongers et al., |991).

Tal índice permite monitorear la colonización y posterior sucesión en un ecosistema después de una perturbación. Se basa en la ubicación de los taxones de nematodos en una escala c-p, que va desde I (estrategas $r$ ) hasta 5 (estrategas K). Los nematodos en el extremo inferior de la escala se consideran colonizadores, oportunistas de ambientes enriquecidos, y por lo tanto indican disponibilidad de nutrientes. Los nematodos en el extremo superior de la escala son persistentes, e indican estabilidad del ecosistema y redes tróficas complejas. Los géneros y especies de una misma familia tienen el mismo valor c-p, excepto en algunas especies marinas (Ferris y Bongers, 2009).

También los triángulos c-p son una forma gráfica de representar la composición de la comunidad de nematodos. Con este análisis se diferencia la fauna basal o grupos c-pl y c-p2, indicadores de enriquecimiento, de los grupos c-p3-5, indicadores de estructura (Goede et al., 1993).

En fechas más recientes se han desarrollado modelos indicadores de las vías metabólicas en el ecosistema del suelo, que pueden ser aplicados convenientemente a través de grupos funcionales de los nematodos. Los índices de basicidad, estructura, enriquecimiento y vías metabólicas de las redes tróficas son indicadores de los procesos en el ecosistema. Estos índices combinan los grupos tróficos a los que pertenecen los diferentes taxones de nemátodos con su grupo c-p en una matriz, la cual describe la condición de enriquecimiento y estructura de un ecosistema (Ferris et al., 200 I). 
Los índices de diversidad trófica y de madurez, con sus variaciones, pueden diferenciar la condición ecológica del suelo en una escala regional mejor de lo que lo hacen géneros en particular. Se ha determinado que la utilidad de las comunidades de nematodos como bioindicadores de la condición del ecosistema mejora si se utiliza una serie de índices que se complementen entre sí (Neher, 200 I).

En el año 2003, cuatro sistemas agrícolas (café orgánico, tomate orgánico, café convencional y tomate convencional) y uno natural (bosque secundario de sucesión avanzada), situados en San Ramón de Alajuela, fueron muestreados con el objetivo de estudiar la composición de la comunidad de nematodos en cada uno de ellos.

En el cuadro I se muestran las variables calculadas para la comunidad de nemátodos en cada uno de los ecosistemas. Entre las variables se incluyen índices de diversidad y madurez.

Estos resultados muestran el cambio en las comunidades de nematodos y la utilidad de su análisis para evaluar el estado de los ecosistemas y el efecto de las alteraciones en estos. En primer lugar, se observa cómo el cultivo y las actividades agrícolas provocan la disminución en la abundancia de nematodos en el suelo, tendencia que ya ha sido observada en otros estudios (Hodda et al., 1997).

En cuanto a la diversidad (índices de diversidad y de equidad), se observan pocas diferencias entre los sistemas. Aunque algunos estudios indican que las alteraciones pueden provocar el descenso en el valor del índice de diversidad, otros reportan poca o ninguna diferencia entre sistemas alterados y no alterados (Neher y Campbell, 1994, Neher, 1995).

Cuadro I.Variables calculadas para la comunidad de nematodos en cuatro sistemas agrícolas y uno natural en San Ramón de Alajuela.

\begin{tabular}{|c|c|c|c|c|c|}
\hline & Bosque & Café orgánico & Café convencional & Tomate orgánico & Tomate convencional \\
\hline $\begin{array}{c}\text { Breve descripción del } \\
\text { ecosistema }\end{array}$ & $\begin{array}{c}\text { Bosque } \\
\text { secundario } \\
\text { de sucesión } \\
\text { avanzada }\end{array}$ & $\begin{array}{c}\text { Plantación } \\
\text { de café con } \\
\text { sombra de árboles. } \\
\text { Poco manejo } \\
\text { agronómico. }\end{array}$ & $\begin{array}{l}\text { Monocultivo de } \\
\text { café. } \\
\text { Fertilización y } \\
\text { control de plagas } \\
\text { con agroquímicos } \\
\text { sintéticos. }\end{array}$ & $\begin{array}{c}\text { Monocultivo } \\
\text { de tomate. } \\
\text { Fertilización con } \\
\text { compost y materia } \\
\text { orgánica. }\end{array}$ & $\begin{array}{l}\text { Monocultivo de } \\
\text { tomate. Fertilización } \\
\text { y control de plagas } \\
\text { con agroquímicos } \\
\text { sintéticos. }\end{array}$ \\
\hline $\begin{array}{l}\text { Nemátodos/ } \mathrm{m}^{2} \text { de } \\
\text { suelo }\end{array}$ & $2,52 \times 10^{6}$ & $1,81 \times 10^{6}$ & $9,46 \times 10^{5}$ & $2,10 \times 10^{6}$ & $9,07 \times 10^{5}$ \\
\hline $\begin{array}{l}\text { Diversidad Shannon- } \\
\text { Wiener de familias.' }\end{array}$ & 4,05 & 3,23 & 4,23 & 3,63 & 2,83 \\
\hline Equidad de familias ${ }^{2}$ & 0,58 & 0,56 & 0,46 & 0,48 & 0,43 \\
\hline Índice de madure $z^{3}$ & 3,17 & 2,41 & 2,35 & 1,96 & $|, 6|$ \\
\hline $\begin{array}{c}\text { micófagos/ } \\
\text { bacteriófagos }{ }^{4}\end{array}$ & 0,92 & 0,20 & 0,51 & 0,32 & 0,37 \\
\hline
\end{tabular}

I. Diversidad Shannon-Wiener de familias: se calculó como: $\mathrm{Ht}=-\Sigma$ tilog ${ }_{2}$ i donde $t_{i}$ es la proporción del total de la muestra que corresponde al taxón i. Combina dos componentes de la diversidad: el número de especies y la igualdad o desigualdad de la distribución de individuos en las diversas especies.

2. Equidad de familias: se calculó como: $\mathrm{Et}=\mathrm{Ht} / \mathrm{Ht}_{\text {máx }}$ dónde $\mathrm{Ht}_{\text {máx }}=\log _{2}$ (número de taxones). Compara la diversidad calculada con la diversidad máxima posible, no es una medida influenciable por los taxones abundantes o por la cantidad de taxones como sí lo es el índice de diversidad.

3. Índice de madurez (Bongers, 1990): se calculó como: $M I=S v_{i} p_{i}$, donde $v_{i}$ es el valor c-p en el taxón i y $\mathrm{p}_{i}$ es la proporción del taxón en la muestra; se excluyen los nemátodos fitófagos. Determina el grado de sucesión alcanzado por un ecosistema y se basa en la ubicación de las familias de nemátodos en cinco grupos c-p que representan diferentes estrategias de supervivencia y requerimientos ecológicos.

4. La relación micófagos/bacteriófagos (Freckman y Ettema, 1993) permite comparar la vía que sigue la descomposición y mineralización de los nutrientes y la producción primaria en el ecosistema. 
Esto se debe a que los cambios son más evidentes en la composición de la comunidad que en su diversidad. El sistema bosque, aunque no tuvo el mayor índice de diversidad, sí muestra el valor más alto de equidad, lo que indica una mejor distribución del total de los individuos en las familias presentes.

El índice de madurez refleja de mejor forma los cambios en el ecosistema. El bosque, que es un sistema no alterado, tiene el mayor índice, mientras que los sistemas con cultivo perenne (café) tienen mayores índices que los cultivos anuales (tomate). Además, los sistemas manejados orgánicamente tuvieron valores más altos que los respectivos cultivos convencionales.

También se detectaron menos individuos pertenecientes a las familias Belondiridae, Tylencholaimidae, Dorylaimidae, Aporcelaimidae, Ironidae y Mydonomidae en los sistemas cultivados con respecto al bosque (no se muestran los resultados). Estas familias, cuyos valores c-p son 4 ó 5 , se caracterizan por tener ciclos de vida largos y ser sensibles a los contaminantes y compuestos nitrogenados (Bongers y Bongers, 1998, Tenuta y Ferris, 2004), sustancias que generalmente son adicionadas a los suelos de los sistemas agrícolas.

La relación micófagos/bacteriófagos indica la participación de hongos y bacterias en las vías de descomposición del ecosistema. En el bosque, este índice se acerca más a la unidad, ya que en los sistemas naturales las vías de descomposición no son dominadas por bacterias como lo son en los sistemas de cultivo, en donde se utiliza fertilización constante (Villenave et al., 200 I). Esta tendencia es más evidente en los cultivos orgánicos, en los cuales se utilizan grandes cantidades de materia orgánica en descomposición para la fertilización.

\section{Consideraciones finales}

Aunque en Costa Rica es necesario hacer más investigación en el campo de las comunidades de nematodos, el uso de estos organismos como indicadores ambientales tiene un futuro prometedor, tanto en el monitoreo de ecosistemas en particular como en la validación de las prácticas de mitigación y en los programas de certificación ambiental.

La gran diversidad de los nematodos los hace excelentes indicadores, sin embargo, esto a la vez constituye una limitante. La identificación taxonómica tradicional requiere personal capacitado, y aunque reconocer las familias y algunos géneros de nematodos resulta sencillo con la capacitación adecuada, la identificación a nivel de especie únicamente la realizan expertos en grupos en particular.

La introducción de herramientas moleculares, como complemento de la taxonomía tradicional, ha permitido mejorar el estudio de la diversidad nematológica. En Costa Rica, el Instituto Tecnológico de Costa Rica, la Universidad Nacional y la Universidad de Costa Rica han logrado avances en este campo.

En el Instituto Tecnológico de Costa Rica se ha utilizado PCR-RFLPs de regiones conservadas del ADN ribosómico (I8S, ITS, D2-D3) y mitocondrial (COI) como métodos de identificación de especies de nemátodos, sin embargo, para evaluar comunidades enteras es necesario desarrollar técnicas como la secuenciación de nueva generación, la cual ha sido muy útil en la evaluación de la diversidad de organismos procariotas (Ferris et al., 20I2).

\section{Bibliografía}

Bongers, T. (1985). Dutch forest nematodes. Nematologica 31, 356-357.

Bongers, T. (1990). The maturity index: an ecological measure of environmental disturbance based on nematode species composition. Oecología 83, 14-19.

Bongers, T., Alkemade, R. \& Yeates, G.W. (1991). Interpretation of disturbance induced maturity decrease in marine nematode assemblages by means of the Maturity Index. Marine Ecology Progress Series 76, I 35- 142.

Bongers, T. \& Bongers, M. (1998). Functional diversity of nematodes. Applied Soil Ecology 10, 239-25I.

Bonger,T. (1999). The maturity index, the evolution of nematode life history traits, adaptive radiation and $\mathrm{cp}$-scaling. Plant and Soil 212, 13-22.

Culman, S.W., Young-Mathews, A., Hollander, A.D., Ferris, $H_{\text {., }}$ Sanchez-Moreno, S., O 'Geen, A.T. \& Jackson, L.E. (20l0). Biodiversity is associated with indicators of soil ecosystem functions over a landscape gradient of agricultural intensification. Landscape Ecology 25, I333-1 348.

Dalea, V.H. \& Beyerler, S.C. (200I). Challenges in the development and use of ecological indicators. Ecological Indicators I ( 1$): 3-10$.

Eisenhauer, N., Cesarz, S., Koller, R., Worm, K. \& Reich, P.B. (20।2). Global change belowground: impacts of elevated $\mathrm{CO}_{2}$, nitrogen, and summer drought on soil food webs and biodiversity. Global Change Biology 18, 435-447. 
European Environment Agency (EEA). (1999). Environmental Indicators: Typology and overview. Copenhagen.

European Environment Agency (EEA). (20I2). Environmental Indicator Report. Ecosystem resilience and resource efficiency in a green economy in Europe. Copenhagen.

Ferris, H., Bongers, T. \& Goede, R.G.M. de. (200I). A framework for soil food web diagnostics: extension of the nematode faunal analysis concept. Applied soil ecology 18, I3-29.

Ferris, H. \& Bongers, T. (2009). Indices developed specifically for analysis of nematode assemblages. En: Wilson, M.J. \& Kakouli-Duarte, T. (Eds.). Nematodes as environmental indicators (pp. 124- | 45). CAB International, Wallingford, UK.

Ferris, H. (20।0). Contribution of nematodes to the structure and function of the soil food web. Journal of Nematology 42(1), 63-67.

Ferris, H., Griffiths, B.S., Porazinska, D.L., Powers, T.O., Wang, K.-H. \& Tenuta, M. (20I2). Reflections on plant and soil nematode ecology: past, present and future. Journal of Nematology 44(2): I I5- 126.

Freckman, D. \& Ettma, C.H. (1993). Assessing nematode communities in agroecosystems of varying human intervention. Agriculture, Ecosystems and Environment 45, 239-26I.

Goede, R.G.M. de, Bongers, T. \& Ettema, C. (1993). Graphical presentation and interpretation of nematode community structure: C-P triangles. Mededlingen Faculteit Landbouwwetenschappen Universiteit Gent, 58, 743-750.

Hawksworth, D.L., Iturriaga, T. \& Crespo, A. (2005). Líquenes como bioindicadores inmediatos de contaminación y cambios medio ambientales en los trópicos. Revista Iberoamericana de Micología 22(2), 71-82.

Hodda, M., Bloemers, G.F., Lawton, J.H. \& Lambshead, P.J.D. (1997). The effects of clearing and subsequent land-use on abundance and biomass of soil nematodes in tropical forest. Pedobiología 4I, 279-294.

Jensen, H.J. \& Mulvey, R.H. (1968). Predaceous nematodes (Mononchidae) of Oregon. Oregon State Monography, 12, I-57.

Neher, D.A. \& Campbell, C.L. (1994). Nematode communities and microbial biomass in soils with annual and perennial crops. Applied Soil Ecology I, 17-28.
Neher, D.A. (1995). Biological diversity in soils of agricultural and natural ecosystems. En: Olson, R., Francis, C. \& Kaffka, S. (Eds.). Exploring the Role of Diversity in Sustainable Agriculture (pp. 55-72). Alliance of crop, soil, and environmental science societies.

Neher, D.A. (200I). Role of nematodes in soil health and their use as indicators. Journal of Nematology 33, 161-168.

Neher, D.A. \& Darby, B.J. (2009). General community indices that can be used for analysis of nematode assemblages. En: Wilson, M.J. \& Kakouli-Duarte, T. (Eds.). Nematodes as environmental indicators (pp. 107-123). CAB International, Wallingford, UK.

Paerl, H.W., Dyble, J., Moisander, P.H., Noble, R.T., Piehler, M.F., Pinckney, J.L., Steppe, T.F., Twomey, L. \& Valdes, L.M. (2003). Microbial indicators of aquatic ecosystem change: current applications to eutrophication studies. FEEMS Microbiology Ecology 46(3): 233-246.

Sánchez-Moreno, S., Nicola, N.L., Ferris, H. \& Zalom, F. (2009). Effects of agricultural management on nematode-mite assemblages: Soil food web indices as predictors of mite community composition. Applied Soil Ecology 4I, I07-I 17.

Tenuta, M. \& Ferris, H. (2004). Sensitivity of nematode life-history groups to ions and osmotic tensions of nitrogenous solutions. Journal of Nematology 36, 85-94.

Villenave, C., Bongers, T., Ekschmitt, K., Djigal, D. \& Chotte, J.L. (200I). Changes in nematode communities following cultivation of soils after fallow periods of different length. Applied Soil Ecology 17, 43-52.

Wasilewska, L. (1994). The effect of age meadows on succession and diversity in soil nematode communities. Pedobiología $38,1-11$.

Yeates, G.W. \& Bongers, T. (1999). Nematode diversity in agroecosystems. Agriculture, Ecosystems and Environment 74, | |3-135.

Yeates, G.W. \& Boag, B. (2006). Female size shows similar trends in all clades of the Phylum Nematoda. Nematology 8, I I I127.

Yeates, G.W., Ferris, H., Moens, T. \& Van der Putten, W.H. (2009). The role of nematodes in ecosystems. En: Wilson, M.J. \& Kakouli-Duarte, T. (Eds.). Nematodes as environmental indicators (pp. I-44). CAB International, Wallingford, UK. 